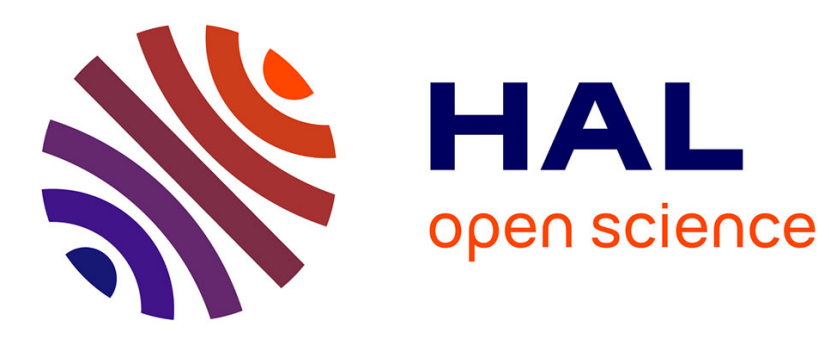

\title{
Localized time-dependent perturbations in metals
}

\author{
Annie Blandin, D. Hone, A. Nourtier
}

\section{To cite this version:}

Annie Blandin, D. Hone, A. Nourtier. Localized time-dependent perturbations in metals. Journal de Physique Lettres, 1975, 36 (4), pp.109-111. 10.1051/jphyslet:01975003604010900 . jpa-00231160

\section{HAL Id: jpa-00231160 https://hal.science/jpa-00231160}

Submitted on 1 Jan 1975

HAL is a multi-disciplinary open access archive for the deposit and dissemination of scientific research documents, whether they are published or not. The documents may come from teaching and research institutions in France or abroad, or from public or private research centers.
L'archive ouverte pluridisciplinaire HAL, est destinée au dépôt et à la diffusion de documents scientifiques de niveau recherche, publiés ou non, émanant des établissements d'enseignement et de recherche français ou étrangers, des laboratoires publics ou privés. 


\title{
LOCALIZED TIME-DEPENDENT PERTURBATIONS IN METALS
}

\author{
A. BLANDIN and D. HONE $\left({ }^{*}\right),\left({ }^{1}\right),(+)$ and A. NOURTIER \\ Laboratoire de Physique des Solides $\left({ }^{2}\right)$, \\ Université Paris-Sud, Centre d'Orsay, 91405 Orsay, France
}

(Reçu le 10 janvier 1975, révisé le 3 février 1975, accepté le 7 février 1975)

\begin{abstract}
Résumé. - La méthode introduite par Keldysh pour traiter les problèmes hors d'équilibre est appliquée au cas de fortes perturbations localisées dépendant du temps dans les métaux, perturbations observées dans divers phénomènes de surface.
\end{abstract}

\begin{abstract}
Methods introduced by Keldysh to treat non-equilibrium problems are applied to strong localized time-dependent perturbations in metals, such as those that arise in various surface phenomena.
\end{abstract}

There are many interesting problems in metals which involve strong local time-dependent perturbations : X-ray absorption edges [1], absorption and desorption of atoms on surfaces, etc... The present work was motivated initially by experiments on ionization probabilities of atoms extracted from metals by ionic bombardment [2]. These experiments show that the ejected atoms retain some memory of the bulk dynamics, to an extent controlled by the time necessary to cross the surface, as compared with the characteristic times of charge and spin dynamics in the bulk. A qualitative discussion of these effects has already been given [3], and the problem has been treated within first order perturbation theory using a time-dependent Anderson Hamiltonian [4]. In this model, an atom leaving the surface is described by a time-dependent admixture matrix element $V_{k d}$, which goes to zero outside the metal. Therefore, the model can describe the problem of desorption as well as that of adsorption of atoms on a surface.

Although the mathematical technique is applicable to the Hamiltonian just described; for simplicity, we shall restrict our treatment here to a simpler model, the Anderson Hamiltonian without spin, where only the diagonal extra-orbital energy varies with time :

$$
H=\sum_{k} \varepsilon_{k} n_{k}+\left(\varepsilon_{d}+v(\tau)\right) n_{d}+\sum_{k}\left(V_{k d} c_{k}^{*} c_{d}+c c\right) \text {. }
$$

$\left(^{*}\right)$ Permanent address : Physics Department, U.C.S.B., Santa Barbara, California, U.S.A.

$\left({ }^{1}\right)$ Supported by the National Science Foundation.

$(\dagger)$ S. R. C. Senior Visiting Lecturer at Oxford University during the latter part of this work.

$\left({ }^{2}\right)$ Laboratoire associé au C.N.R.S.
This Hamiltonian might describe approximately the situation at a metal-metal junction : the energy $v$ in equ. (1) then varies across the junction from zero to a constant value. Thus, $v$ is a function of position and becomes a function of time as a consequence of the motion of the impurity.

For a strong time-dependent perturbation, the system is in general far from equilibrium. A general method has been proposed by Keldysh [5] to treat such non-equilibrium situations. Essentially, this method introduces a new time ordering, so as to make possible the use of Wick's theorem and thereby diagrammatic techniques and Dyson's equation. The times are ordered along a contour from $-\infty$ to $+\infty$ and back to $-\infty$. Therefore, one must specify not only the time, but also whether it is on the increasing or decreasing branch of the contour, and the Green's function $\overline{\bar{G}}\left(\tau, \tau^{\prime}\right)$ becomes a $2 \times 2$ matrix labelled by the branches of the times $\tau$ and $\tau^{\prime}$. It is this matrix Green's function which satisfies the Dyson equation :

$$
\overline{\bar{G}}=\overline{\bar{g}}+\overline{\overline{g \Sigma G}}
$$

where the unperturbed Green's function $\overline{\bar{g}}$ and the self-energy $\overline{\bar{\Sigma}}$ are also $2 \times 2$ matrices. In particular, for both times on the increasing branch we have the usual causal Green's functions :

$$
G_{i j}^{c}\left(\tau, \tau^{\prime}\right)=-i\left\langle 0\left|T\left\{c_{i}(\tau), c_{j}^{+}\left(\tau^{\prime}\right)\right\}\right| 0\right\rangle,
$$

we emphasize that $G^{c}$ does not obey a Dyson equation on the time interval $(-\infty,+\infty)$ (this would violate causality). Of the four matrix elements of $\overline{\bar{G}}$, only two are independent and it is convenient to make a 
canonical transformation to give the functions :

$$
G_{i j}^{r}\left(\tau, \tau^{\prime}\right)=-i \theta\left(\tau-\tau^{\prime}\right)\left\langle 0\left|\left\{c_{i}(\tau), c_{j}^{+}\left(\tau^{\prime}\right)\right\}\right| 0\right\rangle
$$

which clearly is a retarded function [6]; $G_{i j}^{a}\left(\tau, \tau^{\prime}\right)$ which is similarly defined as an advanced function; and

$$
F_{i j}\left(\tau, \tau^{\prime}\right)=-i\left\langle 0\left|\left\{c_{i}(\tau), c_{j}^{+}\left(\tau^{\prime}\right)\right\}\right| 0\right\rangle .
$$

$G^{r}$ and $G^{a}$ satisfy standard Dyson equations and

$$
F=g^{r} \Omega G^{a}+g^{r} \Sigma^{r} F+f \Sigma^{a} G^{a} .
$$

Integrations over intermediate times have been suppressed here and $\Omega, \Sigma^{r}, \Sigma^{a}$ are the non-zero components of the transformed self-energy matrix. In particular, for the perturbation described by Hamiltonian (1),

$$
\Omega=0 ; \quad \Sigma^{r}=\Sigma^{a}=v(\tau) \delta\left(\tau-\tau^{\prime}\right) .
$$

For such a one body potential (in a many electron system) $G^{a}$ and $G^{r}$ satisfy simple one particle equations; they do not involve the occupation numbers and thus the existence of a Fermi surface. $G^{a}$ and $G^{r}$ are then readily obtained, in particular, for the Hamiltonian (1). The many-body aspects reside in the function $F$ which is given by iterative solution of equ. (6) as :

$$
F=\left(1+G^{a} v\right) f\left(1+v G^{r}\right) .
$$

Thus, for our simple model, we have an exact solution, in terms of explicit integrals.

Let us consider two limiting special cases :

1. SUDDEN SWITCHING OF $v: v(\tau)=v \theta(\tau)$. Clearly, at long times, the Green's function will be those appropriate to the Hamiltonian (1) with $v=$ constant. One finds for $\left\langle n_{d}(\tau)\right\rangle$ in the long time limit :

$$
\left\langle n_{d}(\tau)\right\rangle \simeq \frac{1}{2}-\frac{1}{\pi} \tan ^{-1} \frac{\varepsilon_{d}+v}{\Delta}=\frac{\eta}{\pi}
$$

where $\Delta$ is the resonance width defined as usual by :

$$
\Delta=\pi \rho\left(\varepsilon_{d}+v\right)\left\langle\left|V_{k d}\right|^{2}\right\rangle
$$

and $\eta$ is the phase shift at the Fermi level corresponding to the potential $\varepsilon_{d}+v$. This limit is approached exponentially at a rate $\Delta$. The exact expression will not be given here.

One can remark that there is no singular term being proportional to $t^{-\alpha}$ as it arises in the $\mathrm{X}$-ray absorption edge [1].

2. SLOWLY VARYING $v(\tau)$. - When the time scale of variation of $v(\tau)$ is large compared with $1 / \Delta$, we can make a systematic expansion of the Green's functions. The leading terms for $n_{d}(\tau)$ are given by :

$$
n_{d}(\tau)=\frac{1}{\pi} \eta(\tau)+\pi \rho_{d}^{2}(\tau) \frac{\mathrm{d} v}{\mathrm{~d} \tau}
$$

where $\eta(\tau)$ is the phase shift at the Fermi level due to the instantaneous potential $\varepsilon_{d}(\tau)=\varepsilon_{d}+v(\tau)$, and $\rho_{d}(\tau)$ is similarly the instantaneous $d$ spectral density at the Fermi level for the same potential. In equ. (10), the first term represents the adiabatic result, corresponding to the potential $v(\tau)$ (Friedel sum rule). The second term exhibits a retardation effect.

The same expansion can be applied to the time variation of the energy. The derivative $\mathrm{d}\langle H(\tau)\rangle / \mathrm{d} \tau$ is just the force on the atom, $F$, multiplied by $(-u)$ where $u$ is the velocity of that atom. If we recall that all the time dependence arises from the motion of the atom, we can regard $v(\tau)=v(x(\tau))$ and $\eta(\tau)$ as functions of position $x$ and $\mathrm{d} v / \mathrm{d} \tau=(\mathrm{d} v / \mathrm{d} x) u$. Using (10) we get the leading terms of the expansion for the force $F$ :

$$
F(x)=-\frac{\eta(x)}{\pi} \frac{\mathrm{d} v}{\mathrm{~d} x}-\frac{1}{\pi}\left(\frac{\partial \eta}{\partial x}\right)^{2} u .
$$

The first term is a conservative force associated with the spatial variation of $\varepsilon_{d}(x)$. The second term is a friction term proportional and opposite in sign to the velocity $u$ of the atom. In the expansion terms would then appear proportional to $u^{2}$ and to the acceleration $\mathrm{d} u / \mathrm{d} t$ of the atom : this last term may be interpreted as a change of the effective mass of the atom moving through the medium.

The friction force has been calculated for the case of an ion moving in a uniform medium [7] : in that case the friction coefficient is proportional, as it should be, to the resistivity. For our simple Hamiltonian that case is described by a varying $V_{k d}$ :

$$
V_{k d}(t)=V_{k d}(0) \mathrm{e}^{i \mathbf{k} \cdot \mathbf{R}(t)}
$$

where $\mathbf{R}(t)$ is the position of the ion at time $t$. Using this time dependence, one recovers the quoted results [7] and a friction term proportional to $\sin ^{2} \eta$. We conclude that there exists two different regimes for the friction.

In summary, we have described a systematic technique for treating strong localized time dependent perturbations [8]. This can be useful for studying the motion of particles in inhomogeneous environments (particularly near surfaces). We have, for example, shown how to calculate friction forces for a particular model Hamiltonian; these forces have usually been treated phenomenologically, though a completely different a priori calculation has been done recently by Suhl and coworkers [9]. The method used here can be extended to other Hamiltonians. The interesting case of a time dependent $V_{k d}$, discussed above, will be published in a forthcoming paper. It will be particularly relevant for the understanding of ionization probabilities, absorption and desorption of atoms on surfaces. 
Finally, we can relate our work to previous studies on time dependent problems. First, the functional integral method effectively replaces two body interactions by a fluctuating one-body potential [10]. However, these fluctuations do not depend on a true time variable; the corresponding Green's functions do obey a Dyson equation (in contrast to $G^{c}$ as discussed above) but they violate causality (in the pseudotime variable). Second, a truly time dependent problem arises in the study of X-ray absorption edges; as discussed by Nozières and de Dominicis [1], [11]. Initially, the Hamiltonian is time-independent and the X-ray response is given in terms of a two particle Green's function. Elimination of deep hole operators introduces an effective one-body potential which is suddenly switched on at time $t$ and off at time $t^{\prime}$.

The X-ray problem is thus related to the calculation of a function $\Phi\left(\tau, \tau^{\prime} ; t t^{\prime}\right)$ which obeys a Dyson equation on the contour $\left(t+\infty ;+\infty t^{\prime}\right)$. It is not a causal function and exhibits singularities which are linked to the infrared catastrophe first pointed by Anderson [12]. These singularities arise because $t^{\prime} \neq t$. In our case on the contrary, $G^{c}\left(\tau, \tau^{\prime}\right)$, which is a causal function, obeys a Dyson equation on the contour $(t+\infty ;+\infty t)$ and does not show up singularities.

Acknowledgments. - The authors would like to acknowledge useful and pleasant discussions with R. Balian and G. Toulouse.

\section{References}

[1] Nozières, P. and De Dominicis, C. T., Phys. Rev. 178 (1969) 1097.

[2] Blaise, G. and Slodzian, G., J. Physique 35 (1974) 237.

[3] Joyes, P. and Toulouse, G., Phys. Lett. 39A (1972) 267. Djafari-Rouhani, B., Joyes, P. et Toulouse, G., J. Physique 34 (1973) 741.

[4] Djafari-Rouhani, B., Thèse de $3^{\mathrm{e}}$ cycle, Orsay (1974).

[5] Keldysh, L. V., Sov. Phys. J.E.T.P. 20 (1965) 1018.

[6] Here we use the standard notation for retarded and advanced functions, which is the opposite of Keldysh's notation

[7] Edwards, S. F., Proc. Phys. Soc. 57 (1965) 469.

Rousseau, J., Stoddart, J. C. and March, N. H., J. Phys. C 5 (1972) 175
[8] Applications to steady state non equilibrium problems have been made, e.g. by

Caroli, C., Combescot, R., Nozières, P. and Saint-James, D., J. Phys. C 4 (1971) 916 for a direct calculation of the tunneling current and for photoemission.

[9] D'Agliano, E. G., Schaich, W. L., Kumar, P. and Suhl, H., Nobel 24 (1973) 200, Collective properties of physical systems.

[10] Hamann, D. R., Phys. Rev. B 2 (1970) 1373.

[11] The calculation of related quantities has been carried out by Müller-Hartmann, E., Ramakrishnan, T. V. and TouLOUSE, G., Phys. Rev. B 3 (1971) 1102.

[12] Anderson, P. W., Phys. Rev. Lett. 18 (1967) 1049. 